\title{
Late-Onset Neurodegeneration with Brain Iron Accumulation with Diffusion Tensor Magnetic Resonance Imaging
}

\author{
Syed Omar Shah ${ }^{a}$ Hasit Mehta ${ }^{b}$ Robert Fekete $^{a}$ \\ ${ }^{\mathrm{a}}$ Department of Neurology, New York Medical College, and ${ }^{\mathrm{b}}$ Department of \\ Radiology, Westchester Medical Center, Valhalla, N.Y., USA
}

\section{Key Words}

Neurodegeneration with brain iron accumulation · Neuroferritinopathy · Diffusion tensor imaging $\cdot$ Hyperkinetic movement disorders

\begin{abstract}
Introduction: Neuroferritinopathy is an autosomal dominant neurodegenerative disorder that includes a movement disorder, cognitive decline, and characteristic findings on brain magnetic resonance imaging (MRI) due to abnormal iron deposition. Here, we present a lateonset case, along with diffusion tensor imaging (DTI).

Case Presentation: We report the case of a 74-year-old Caucasian female with no significant past medical history who presented for evaluation of orofacial dyskinesia, suspected to be edentulous dyskinesia given her history of ill-fitting dentures. She had also developed slowly progressive dysarthria, dysphagia, visual hallucinations as well as stereotypic movements of her hands and feet.

Results: The eye-of-the-tiger sign was demonstrated on T2 MRI. Increased fractional anisotropy and $\mathrm{T} 2$ hypointensity were observed in the periphery of the globus pallidus, putamen, substantia nigra, and dentate nucleus. T2 hyperintensity was present in the medial dentate nucleus and central globus pallidus.

Discussion: The pallidal MRI findings were more typical of pantothenate kinase-associated neurodegeneration (PKAN), but given additional dentate and putamenal involvement, lack of retinopathy, and advanced age of onset, PKAN was less likely. Although the patient's ferritin levels were within low normal range, her clinical and imaging features led to a diagnosis of neuroferritinopathy.

Conclusion: Neurodegeneration with brain iron accumulation (NBIA) is a rare cause of orofacial dyskinesia. DTI MRI can confirm abnormal iron deposition. The location of abnormal iron deposits helps in differentiating NBIA subtypes. Degeneration of the dentate and globus pallidus may occur via an analogous process given their similar T2 and DTI MRI appearance.
\end{abstract}




\section{Introduction}

Neuroferritinopathy is an autosomal dominant neurodegenerative disorder that is grouped within a family of disorders called neurodegeneration with brain iron accumulation (NBIA). The disease most often includes a movement disorder, cognitive decline, and typical magnetic resonance imaging (MRI) appearance. This disease develops from mutations in the ferritin light chain gene (FTL1) [1]. Seven known mutations of this gene have been discovered [2]. The resulting mutations alter the reading frame and disrupt the ferritin light chain polypeptide's tertiary structure, leading to inappropriate release from ferritin polymers [3]. These effects are primarily seen in the central nervous system. The resulting mutations will cause a low serum ferritin level in most patients [2].

A variety of abnormal, involuntary movements is demonstrated in the clinical presentation of neuroferritinopathy. Published clinical descriptions include orofacial dyskinesia, focal dystonia, chorea, finger and toe stereotypies, parkinsonism, and tremors $[1,4-6]$. Although it is an autosomal disease, phenotypic variations have been described [1]. In contrast to other NBIA disorders, pigmentary retinopathy and acanthocytosis have not been reported in neuroferritinopathy [2].

Abnormalities seen on MRI initially include iron deposition, and subsequently edema, gliosis, cystic changes, and cortical atrophy $[1,4]$. Central nervous system iron deposition occurs with normal aging, especially in the globus pallidus, putamen, substantia nigra, and dentate. Patients affected with neuroferritinopathy demonstrate iron and ferritin deposits in excess of expected age-related accumulation, most prominently within the basal ganglia [1,5]. Cystic changes are most often pronounced within the globus pallidus and putamen [2], but may occur in the thalamus, dentate, and caudate $[1,4]$. Sometimes MRI also demonstrates cortical atrophy, both within the cerebellum and cerebral cortices [2]. Overall, there is a progression of MRI changes from T2 hypointensities due to iron deposition to T2 hyperintensities likely caused by the start of cystic degeneration. Here, we present a late-onset case, along with diffusion tensor imaging (DTI).

\section{Case Presentation}

A 74-year-old, right-handed Caucasian female presented for evaluation of involuntary mouth movements. Her teeth had been removed 15 years ago and were replaced with dentures. Initially, there were no involuntary movements of the mouth or tongue. She had stopped wearing dentures 5 years prior to presentation, secondary to cost for replacement dentures. Over the past few years, she reported a progressively worsening mouth and tongue dyskinesia, which became bothersome about 1 year ago. She complained that eating would worsen these dyskinesias and, on some occasions, food would fall out of her mouth. She denied any other abnormal movements throughout the rest of her body except for occasional toe movements that would occur bilaterally and involuntarily. The patient also complained of a mildly unsteady gait during the past year. Her previous medical history was significant for anxiety. We cannot rule out a family history as she last had contact with her father when he was 38 years old.

Neurologically, she had no evidence of tremor, myoclonus, or dystonia. There was, however, continuous orofacial dyskinesia (see supplementary video). In the setting of her ill-fitted dentures, this was initially believed to be edentulous dyskinesia [7, 8]. She also had persistent stereotypic movements of her hands and feet bilaterally. Additionally, there was motor impersistence as the patient was unable to protrude her tongue for longer than $6 \mathrm{~s}$. On cranial nerve testing, the patient 
demonstrated saccadic pursuit and mild delay in saccade initiation. Her gait was somewhat widebased but with normal initiation, arm swing, and stride length. The remainder of her exam was noncontributory and without any deficits in motor or sensory examination.

A detailed history was taken, including all medications the patient had received in her lifetime. The patient denied ever being on any type of neuroleptics or antipsychotic medications, including haloperidol. Given that the patient presented with saccadic dysfunction as well as bilateral extremity sterotypies, a broad differential diagnosis of hyperkinetic movement disorders was considered. The patient was sent for a CT scan in search of hyperdense calcifications due to the phenotypic similarity of Fahr's Syndrome. In addition, MRI along with DTI scans were performed.

\section{Results}

A review of her complete blood count demonstrated no acanthocytes, excluding chorea-acanthocytosis [9]. Given imaging and clinical features discussed below, neuroferritinopathy was considered. Serum ferritin is a useful initial test for neuroferritinopathy since it is usually low in these patients. The ferritin level in our patient was $50 \mu \mathrm{g} / \mathrm{l}$. A recent review of all published cases of neuroferritinopathy noted that after using a cutoff of $30 \mu \mathrm{g} / \mathrm{l}, 64 \%$ of the males and $84 \%$ of the females had low ferritin levels. Presence of low ferritin is not necessary in the diagnosis of neuroferritinopathy [10].

Hypodensities consistent with iron deposition instead of expected hyperdensities from calcium deposits in Fahr's syndrome were seen in bilateral basal ganglia on a head CT (online suppl. fig. 1; for all online suppl. material, see www.karger.com/doi/10.1159/000345871). The combination of a T2 hypointense rim and T2 hyperintense center of the globus pallidus (eye-of-the-tiger sign) was present bilaterally (fig. 1). T2 hypodensity of the putamen was also present. Sequences of the midbrain showed a hypointense substantia nigra and red nucleus. A black and white fractional anisotropy (FA) map showed areas of increased anisotropy in the substantia nigra (fig. 2). Analogous findings were seen in the dentate nucleus with a T2 hypointense lateral portion and a T2 hyperintense medial portion, along with increased anisotropy on the FA map within the dentate nucleus ( $\underline{\text { fig. } 3 \text { ). }}$.

\section{Differential Diagnosis}

Given evidence of abnormal iron depositions seen on MRI corroborated by hypodensities seen on the patient's CT scan, NBIA was considered. Perhaps the bestknown disorder among these is pantothenate kinase-associated neurodegeneration (PKAN/NBIA1), formerly known as Hallervorden-Spatz disease in typical young-onset cases and Hallervorden-Spatz syndrome in atypical late-onset cases. Typical PKAN is an early-onset movement disorder due to mutation of the PANK2 gene [11]. Clinically, individuals present with progressive pigmentary retinopathy, severe dystonia, and sometimes with neuropsychiatric features or cognitive decline. Interestingly, acanthocytes may be seen in PANK2. Brain MRI findings include a hypointense outer rim with central hyperintensity of the globus pallidus on $\mathrm{T} 2$ and $\mathrm{T} 2 *$ sequences, known as the eye-of-the-tiger sign [12]. Although thought to be pathognomonic for the PANK2 mutation, the eye-of-the-tiger sign was documented in neuroferritinopathy as well as atypical PKAN [12-14]. Our patient clearly demonstrated this sign, but lacked certain other characteristics typical of PKAN. In most PKAN cases, T2 changes are present only in the globus pallidus and substantia nigra [2]. Our patient's lesions were widespread, 
and along with late onset, lack of progressive pigmentary retinopathy, or severe dystonia, a diagnosis of PKAN was not pursued further.

Although the patient did not demonstrate a low serum ferritin level that would have been more consistent with neuroferritinopathy, her MRI changes along with her presentation of orofacial dyskinesias, upper and lower extremity stereotypies, and lack of pigmentary retinopathy led us to a diagnosis of neuroferritinopathy (NBIA3). There was evidence of iron deposition outside the globus pallidus and substantia nigra, namely in the putamen, red nucleus, and lateral dentate, seen as T2 hypointense areas. In addition, medial dentate $\mathrm{T} 2$ hyperintensity due to cystic degeneration supported neuroferritinopathy.

Another NBIA disorder, aceruloplasminemia, clinically manifests by iron accumulation in the brain, liver, and reticuloendothelial system [2]. Ceruloplasmin gene mutation leads to abnormal iron deposition in this disease [15]. These patients can present with cerebellar ataxia, chorea, facial grimacing, and progressive dementia. There are also cases reported that link this disorder to the development of diabetes mellitus given the widespread iron deposition throughout the body [15]. Iron was not detected in other organs in our patient. MRI findings demonstrated iron deposition in the entire basal ganglia along with thalamic involvement seen on $\mathrm{T} 2$ and $\mathrm{T} 2 *$, compared to neuroferritinopathy in which $15 \%$ of cases are reported to have all nuclei involved, and none with thalamic abnormalities [2,12]. Without the classic clinical picture, along with the absence of thalamic involvement on MRI, aceruloplasminemia was not considered consistent with our patient's condition.

Neuroaxonal dystrophy (NAD/NBIA2) has been well described and attributed to mutations in the calcium-independent phospholipase A2 gene PLA2G6. It was initially seen as a disease found in infancy, but there have been individuals who were thought to have idiopathic NBIA with the PLA2G6 mutation [16]. Symptoms in NAD are consistent with severe hypotonia, ataxia, dystonia, and, most notably, peripheral neuropathy.

A recent addition to the NBIA group of disorders is fatty acid hydroxylase-associated neurodegeneration (FAHN), which is a result of mutations in the fatty acid-2 hydroxylase FA2H gene [17]. Patients are usually affected earlier in life compared to neuroferritinopathy patients and present with spastic quadriparesis, ataxia, and dystonia [17]. MRI demonstrates of iron accumulation with T2 hypointensities in the globus pallidus and pontine, cerebellar atrophy as well as T2 white matter hyperintensities [17].

Another novel NBIA syndrome, Woodhouse-Sakati Syndrome (WSS), has been described and is the result of a single base pair deletion in the c2orf37 gene [18]. Most cases have been reported from families of Middle Eastern origin. Symptoms include diabetes mellitus, choreoathetosis, and dystonia [19]. The hallmark feature of this disease is alopecia. MRI studies demonstrate globus pallidus T2 hypointensities consistent with the NBIA group of disorders [18]. To our knowledge, there has been no eye-of-the-tiger sign seen on MRI in either FAHN or WSS. Because our patient presented later in life, without pontocerebellar atrophy and complaints of alopecia, a diagnosis of either FAHN or WSS was not supported. Juvenile amyotrophic lateral sclerosis presentation of NBIA due to c19orf12 mutation (NBIA4) was not considered [20]. 


\section{Discussion}

The approach to the patient's diagnosis was stepwise in fashion, and we believe this allowed us to come to the proper diagnosis. The patient's initial symptoms were consistent with edentulous dyskinesia secondary to ill-fitting dentures. With regard to her other extrapyramidal findings, a CT scan was indicated due to concerns about Fahr's disease. The CT scan, however, demonstrated a surprisingly different picture given the hypodensities seen. This led us to request the MRI that demonstrated the eyeof-the-tiger sign along with the multiple $\mathrm{T} 2$ changes noted above. The eye-of-the-tiger sign can lead to a diagnosis of PKAN, but as mentioned, this sign is no longer pathognomonic for the PANK2 gene and has been previously described in neuroferritinopathy.

MRI imaging reveals multiple stages of neuroferritinopathy. When T2* imaging is performed with a gradient echo sequence, the results are very sensitive to changes in paramagnetism that is seen with iron deposition [2]. Hence, initial MRI changes are observed with $\mathrm{T} 2 *$ imaging as hypointensities within the basal ganglia. On T2 conventional imaging, minor low-signal hypointensity is seen [21]. As the disease progresses, the $\mathrm{T} 2 *$ signal loss and the $\mathrm{T} 2$ hypointensity become more pronounced. Eventually, the cerebral cortex, thalamus, substantia nigra, red and dentate nuclei become involved [2]. The next clinical stage of neuroferritinopathy demonstrates a change to T2 hyperintensities as there will be changes in water content secondary to edema and gliosis. This change is thought to be precystic in origin [22]. At advanced stages of neuroferritinopathy, there are characteristic symmetrical basal ganglia T1 hypointense and T2 hyperintense regions consistent with cystic degeneration [21].

Treatment for neuroferritinopathy has not been well established. Iron chelating agents have been tried due to the accumulation of iron, but they have not been found to be efficacious. A recent review of all therapies listed in case reports [10] demonstrated that 14/15 patients received benefit from tetrabenazine. Lehn et al. [10] also found that benzodiazepines, botulinum toxin chemodenervation, anticholinergics, and muscle relaxants, including baclofen, might be beneficial for symptomatic therapy. Unfortunately, our patient did not respond to the use of amantadine. We were unable to prescribe tetrabenazine secondary to cost. Reserpine, a dopamine depleter which may have provided relief, is no longer available in the USA or Canada. Given her history of visual hallucinations, anticholinergics were avoided.

\section{Conclusion}

Neuroferritinopathy is an autosomal dominant neurodegenerative disorder that is grouped within the family of disorders designated NBIA. The disease will most often include a movement disorder, cognitive decline, and typical MRI appearance. Movements include orofacial dyskinesia, focal dystonia, chorea, finger and toe stereotypies, parkinsonism, and tremors. MRI studies demonstrated a progression from $\mathrm{T} 2$ hypointensities due to iron deposition to T2 hyperintensities likely caused by the start of cystic degeneration. Serum ferritin levels can be low, but this is not pathognomonic for this disease. Treatment is limited but there has been good response to tetrabenazine. 


\section{Disclosure Statement}

Dr. Fekete received honoraria from Medlink, Inc., and serves as a consultant for Teva Neuroscience, Inc. and Lundbeck, LLC.
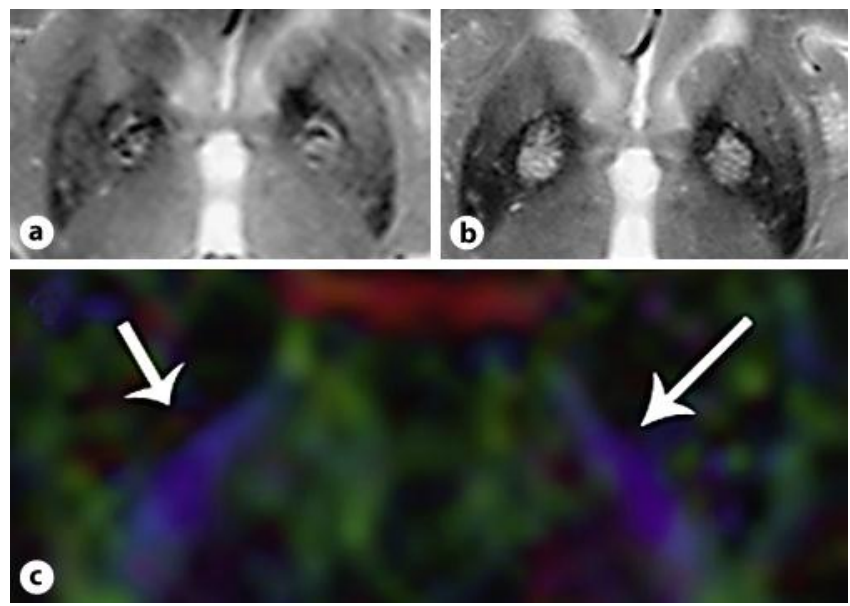

Fig. 1. Eye-of-the-tiger sign is seen on $\mathrm{T} 2 *(\mathrm{a})$ and $\mathrm{T} 2$ (b) sequences. Areas of increased fractional anisotropy corresponding to iron deposition (c) are seen in the right basal ganglia (orange; short arrow) and barely visible on the other side (violet; long arrow).
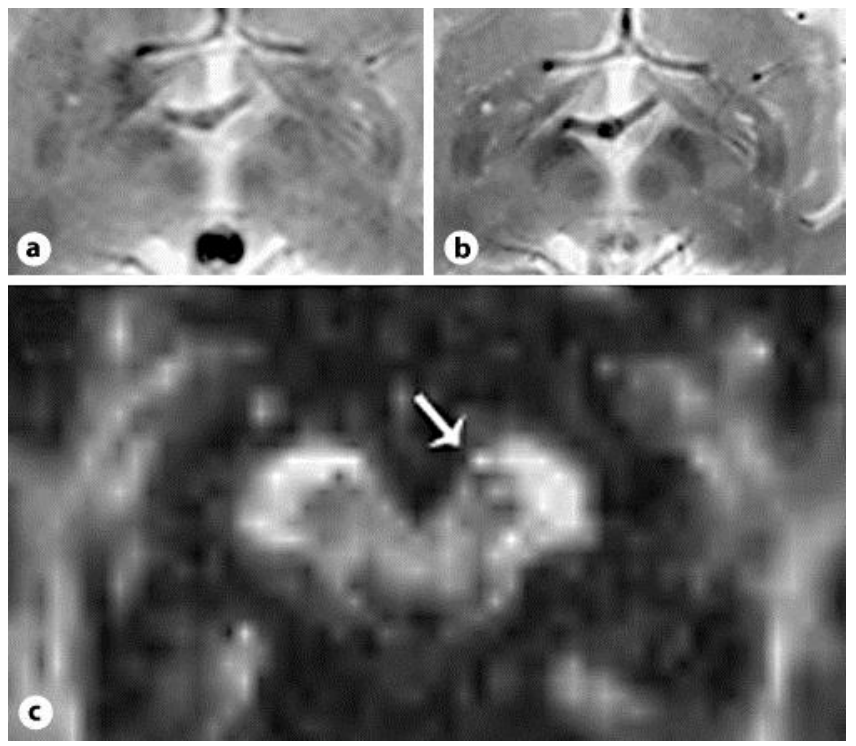

Fig. 2. T2* (a) and T2 (b) sequences of the midbrain showing a hypointense substantia nigra and red nucleus. The black and white FA map (c) shows areas of increased anisotropy in the substantia nigra (arrow). 

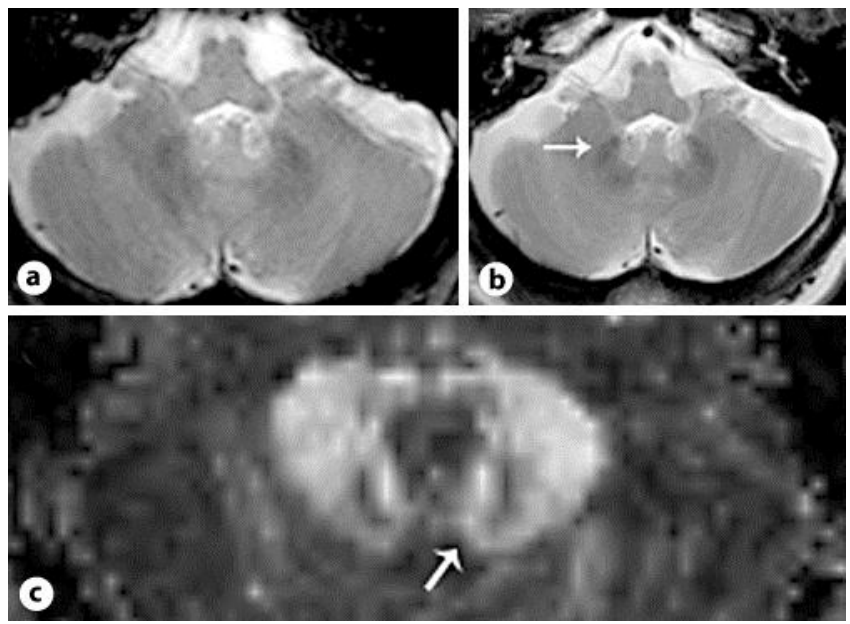

Fig. 3. T2* (a) and T2 (b) sequences of the cerebellum showing hypointense areas of the dentate due to iron deposition (arrow) surrounding hyperintense areas, possibly analogous to the eye-of-thetiger sign of the globus pallidus. The black and white FA map (c) shows increased anisotropy of the dentate nucleus (arrow).

\section{References}

$\rightarrow 1$ Curtis AR, Fey C, Morris CM, et al: Mutation in the gene encoding ferritin light polypeptide causes dominant adult-onset basal ganglia disease. Nat Genet 2001;28:350-354.

-2 Ohta E, Takiyama Y: MRI findings in neuroferritinopathy. Neurol Res Int 2012;2012:197438.

-3 Vidal R, Miravalle L, Gao X, et al: Expression of a mutant form of the ferritin light chain gene induces neurodegeneration and iron overload in transgenic mice. J Neurosci 2008;28:60-67.

4 Vidal R, Ghetti B, Takao M, Brefel-Courbon C, Uro-Coste E, Glazier BS, et al: Intracellular ferritin accumulation in neural and extraneural tissue characterizes a neurodegenerative disease associated with a mutation in the ferritin light polypeptide gene. J Neuropathol Exp Neurol 2004;63:363-380.

5 Mancuso M, Davidzon G, Kurlan RM, Tawil R, Bonilla E, Di Mauro S, Powers JM: Hereditary ferritinopathy: a novel mutation, its cellular pathology, and pathogenetic insights. J Neuropathol Exp Neurol 2005;64:280-294.

6 Devos D, Tchofo PJ, Vuillaume I, et al: Clinical features and natural history of neuroferritinopathy caused by the 458dupA FTL mutation. Brain 2009;132:e109.

7 Koller WC: Edentulous orodyskinesia. Ann Neurol 1983;13:97-99.

8 Blanchet PJ, Rompré PH, Lavigne GJ, Lamarche C: Oral dyskinesia: a clinical overview. Int J Prosthodont 2005; 18:10-19.

9 Levine IM, Estes JW, Looney JM: Hereditary neurological disease with acanthocytosis. A new syndrome. Arch Neurol 1968;19:403-409.

10 Lehn A, Boyle R, Brown H, Airey C, Mellick G: Neuroferritinopathy. Parkinsonism Relat Disord 2012;18:909-915.

11 Zhou B, Westaway SK, Levinson B, Johnson MA, Gitschier J, Hayflick SJ: A novel pantothenate kinase gene (PANK2) is defective in Hallervorden-Spatz syndrome. Nat Genet 2001;28:345-349.

12 McNeill A, Birchall D, Hayflick SJ, et al: T2* and FSE MRI distinguishes four subtypes of neurodegeneration with brain iron accumulation. Neurology 2008;70:1614-1619.

13 Kumar N, Boes CJ, Babovic-Vuksanovic D, Boeve BF: The 'eye-of-the-tiger' sign is not pathognomonic of the PANK2 mutation. Arch Neurol 2006;63:292-293.

14 Strecker K, Hesse S, Wegner F, Sabri O, Schwarz J, Schneider JP: Eye of the tiger sign in multiple system atrophy. Eur J Neurol 2007;14:e1-e2. 
15 Yoshida K, Furihata K, Takeda S, Nakamura A, Yamamoto K, Morita H, Hiyamuta S, Ikeda S, Shimizu N Yanagisawa N: A mutation in the ceruloplasmin gene is associated with systemic hemosiderosis in humans. Nat Genet 1995;9:267-272.

16 Gregory A, Westaway SK, Holm IE, et al: Neurodegeneration associated with genetic defects in phospholipase A(2). Neurology 2008;71:1402-1409.

-17 Kruer MC, Paisán-Ruiz C, Boddaert N, Yoon MY, Hama H, Gregory A, Malandrini A, Woltjer RL, Munnich A, Gobin S, Polster BJ, Palmeri S, Edvardson S, Hardy J, Houlden H, Hayflick SJ: Defective FA2H leads to a novel form of neurodegeneration with brain iron accumulation (NBIA). Ann Neurol 2010;68:611-618.

18 Alazami AM, Al-Saif A, Al-Semari A, et al: Mutations in c2orf37, encoding a nucleolar protein, cause hypogonadism, alopecia, diabetes mellitus, mental retardation, and extrapyramidal syndrome. Am J Hum Genet 2008;83:684-691.

19 Woodhouse NJ, Sakati NA: A syndrome of hypogonadism, alopecia, diabetes mellitus, mental retardation, deafness, and ECG abnormalities. J Med Genet 1983;20:216-219.

-20 Deschauer M, Gaul C, Behrmann C, et al: C19orf12 mutations in neurodegeneration with brain iron accumulation mimicking juvenile amyotrophic lateral sclerosis. J Neurol 2012;259:2434-2439.

21 Crompton DE, Chinnery PF, Bates D, et al: Spectrum of movement disorders in neuroferritinopathy. Mov Disord 2005;20:95-99.

22 Chinnery PF, Crompton DE, Birchall D, et al: Clinical features and natural history of neuroferritinopathy caused by the FTL1 460InsA mutation. Brain 2007;130:110-119. 